\title{
RADIO STRUCTURES OF THE MRC 1-JY SOURCES AND THE UNIFICATION OF RADIO GALAXIES AND QUASARS
}

V. K. KAPAHI ${ }^{1}$, R. M. ATHREYA ${ }^{1}$, C. R. SUBRAHMANYA ${ }^{1}$

${ }^{1}$ NCRA-TIFR, P.Bag 3, Ganeshkhind, Pune-411001, India

P. J. MCCARTHY ${ }^{2}$, W. VAN BREUGEL ${ }^{3}$, J. C. BAKER ${ }^{4}$

${ }^{2}$ OCIW, 813 Santa Barbara St., Pasadena, Ca 91107, USA

3 IGPP, LLNL, Livermore, Ca 94550, USA

${ }^{4}$ MRAO, Madingley Road, Cambridge CB3 OHE, UK

AND

R.W.HUNSTEAD ${ }^{5}$

${ }^{5}$ Astrophy. Dept., University of Sydney, NSW2006, Australia

The viewing angle of $\sim 45^{\circ}$ (between the jet axis and the line of sight) dividing quasars and radio galaxies in the orientation-based unified scheme (Barthel 1989) is based largely on the observation that in the redshift range of $0.5<\mathrm{z}<1.0$ in the low-frequency 3CRR sample, there are about twice as many radio galaxies than quasars and the median linear size of galaxies is about twice that of the quasars. The relative numbers and sizes of quasars in other redshift ranges in the $3 \mathrm{CRR}$ are however in conflict with the simple unified scheme even if the opening angle is considered to evolve with epoch or radio luminosity (Kapahi 1990; Singal 1993). Larger sources samples, preferably selected at low radio frequencies, are clearly important in such statistical studies.

We report here the preliminary results of a comparison of the numbers and sizes of quasars and radio galaxies in a much larger complete sample of MRC radio sources defined by $\mathrm{S}_{408}>0.95 \mathrm{Jy}$ and $-30^{\circ}<\delta<-20^{\circ}$, for which we have made VLA radio maps, near-complete optical identifications and are attempting to obtain spectroscopic redshifts (Kapahi et. al, 1996). Of the 558 sources in our sample we have defined a complete sample of 105 quasars (with $95 \%$ spectroscopic redshifts) and 447 radio galaxies (with $60 \%$ measured redshifts and $22 \%$ estimated from $\mathrm{K}$ magnitudes). 


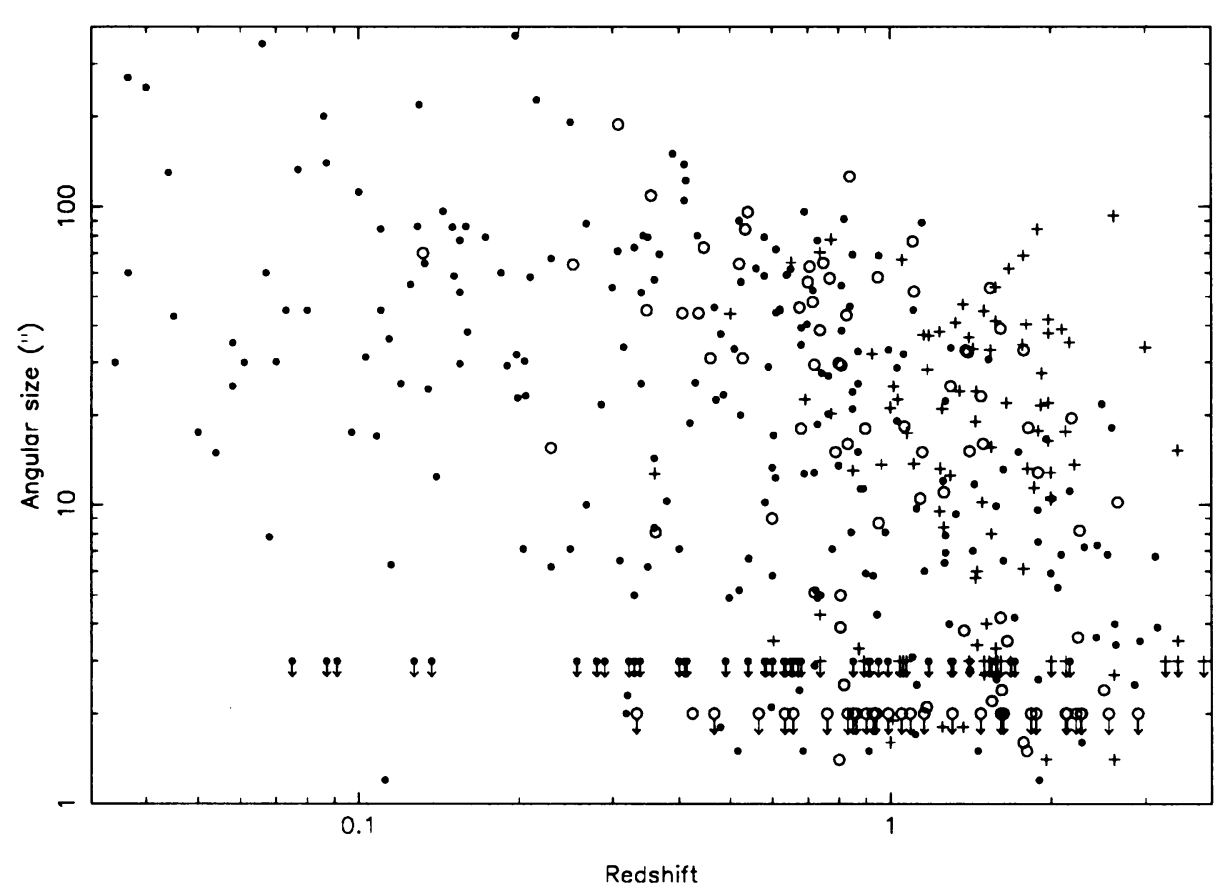

Figure 1. The angular size - redshift plot for radio galaxies (' $\bullet$ ' measured $\mathrm{z}$, '十' estimated $\mathrm{z})$ and quasars (0) with known $\mathrm{z}$ in the MRC 1-Jy samples.

The angular size - redshift plot for all the quasars and galaxies with known or estimated redshifts is shown in Figure 1. Surprisingly, we see no systematic difference between galaxies and quasars in either the maximum angular sizes or the range of angular sizes, contrary to the prediction of the unified model which requires the projected linear sizes of quasars to be almost a factor of two smaller than for galaxies. The medium linear sizes of galaxies and quasar in the redshift ranges of 0.25 to $0.5,0.5$ to 1.0 and 1.0 to 2.0 do not, in fact, show any significant difference. There is also no systematic change in the quasar fraction as a function of redshift. Our new sample thus provides little evidence for any epoch or luminosity dependence of the torus opening angle and the data are hard to understand in the simple orientation based unification of quasars and radio galaxies.

\section{References}

Barthel, P.D. (1989) Ap.J., Vol.336, 606.

Kapahi, V.K. (1990) in 'Parsec-scale Radio Jets' (Eds. J.A.Zensus \& T.J.Pearson), Cambridge Univ. Press, p304.

Kapahi, V.K. et al. (1990) The MRC 1-Jy sample of Radio Galaxies - This volume.

Singal, A.K. (1993) MNRAS, Vol.263, 139. 